\title{
gु \\ Rigorous construction of ground state correlations in graphene: Renormalization of the velocities and Ward identities
}

\author{
Alessandro Giuliani* \\ Università di Roma Tre, L.go S. L. Murialdo 1, 00146 Roma, Italy \\ Vieri Mastropietro ${ }^{\dagger}$ \\ Università di Roma Tor Vergata, Viale della Ricerca Scientifica, 00133 Roma, Italy
}

(Received 3 March 2009; published 20 May 2009)

\begin{abstract}
We consider the two-dimensional Hubbard model on the honeycomb lattice, as a model for single-layer graphene with screened Coulomb interactions; at half filling and weak coupling, we construct its ground-state correlations by a convergent multiscale expansion, rigorously excluding the presence of magnetic or superconducting instabilities or the formation of a mass gap. The Fermi velocity, which can be written in terms of a convergent series expansion, remains close to its noninteracting value and turns out to be isotropic; as a consequence, the Dirac cones are isotropic at low energies. On the contrary, the interaction produces an asymmetry between the two components of the charge velocity, in contrast to the predictions based on relativistic or continuum approximations.
\end{abstract}

DOI: 10.1103/PhysRevB.79.201403

PACS number(s): 71.10.-w, 05.10.Cc, 05.30.Fk

The recent experimental realization of a monatomic graphitic film, ${ }^{1}$ known as graphene, has elicited an enormous interest in the study of the properties of two-dimensional (2D) electron systems on the honeycomb lattice, which is the typical underlying structure displayed by single-layer graphene sheets. Graphene is quite different from most conventional quasi-two-dimensional electron gases because of the peculiar quasiparticle dispersion relation, which closely resembles the one of massless Dirac fermions in $2+1$ dimensions. ${ }^{2}$ Already in the absence of interactions, the system displays highly unusual features, such as the anomalous dependence of the cyclotron mass on the electronic density, an anomalous integer-quantum Hall effect, and the insensitivity to localization effects generated by disorder. In real systems, despite the unavoidable presence of electronelectron interactions, such consequences of the relativisticlike dispersion relation have been experimentally verified; 3,4 the observation based on angle-resolved photoemission spectroscopy ${ }^{5,6}$ is compatible with linear dispersion relation and isotropic Fermi velocity.

A basic model for investigating the effect of the electronelectron interactions in graphene is the 2D Hubbard model on the honeycomb lattice, in the presence either of a short or of a long-range interaction, corresponding to the cases of screened or unscreened Coulomb interactions, respectively; see Refs. 7-16. Usually the analysis of this model is performed by mean-field and renormalization-group (RG) methods at the lowest perturbative orders, ${ }^{7-16}$ neglecting the presence of the lattice and replacing the exact dispersion relation by its linear approximation around the Dirac points. However, in such analyses there is no control of the effects produced by the truncations of the exact RG equations or by the considered approximations so that nonperturbative effects, such as a mass generation, have not been excluded so far and are still subject of an active debate. ${ }^{17-21}$ Moreover, possible symmetry-breaking effects due to the presence of the underlying lattice have not been considered so far, and it remains to be seen whether the fact that Lorentz invariance is explic- itly broken by the lattice can induce anisotropic renormalizations of the Dirac cones, or of the spin and charge velocities.

It would be desirable to substantiate the predictions of theoretical analysis by rigorous results and exact solutions and, in the case of controversial issues, to be able to rigorously exclude one conclusion or the other. Unfortunately, there are very few rigorous results about the structure of the ground state of the Hubbard model in two or more dimensions, among which we would like to mention the results in Ref. 22, guaranteeing the uniqueness of the ground state and the vanishing of its total spin. However, as far as we know, so far no results were proved about the existence or nonexistence of long-range order and about the long-distance behavior of correlation in the Hubbard model on the honeycomb or other lattices.

Two theorems reporting the first rigorous construction of the ground-state correlations in the 2D Hubbard model on the honeycomb lattice at half filling, weak coupling, and short-range interactions are stated here. Our results exclude the presence of magnetic or superconducting instabilities and the formation of a mass gap. The interaction changes by a finite amount the wave-function renormalization, and the Fermi velocity. Note that the interacting Fermi velocity remains isotropic, even though the model breaks the invariance under $90^{\circ}$ rotations; the isotropy of the Fermi velocity implies the isotropy of the Dirac cones at low energies.

On the contrary, we predict that the charge velocity develops an asymmetry between the two components, an effect that is, in principle, accessible to experiments. Note that the latter conclusion is in contrast to the naive expectation that weak short-range interactions, being irrelevant in the RG sense, do not alter the Dirac spectrum and the spin and charge velocities. In the case of unscreened Coulomb interactions, we are not yet able to control the convergence of the renormalized series; however, even in this case, we predict that the Fermi velocity remains isotropic at all orders, while the isotropy of the charge velocity is broken already at second order, as in the case of short-ranged interactions. 
Our analysis is based on the methods of constructive renormalization, which have already proven to be quite effective in analyzing one-dimensional (1D) interacting fermionic systems in their ground state ${ }^{23}$ and $2 \mathrm{D}$ systems up to exponentially small temperatures or in the presence of a nonsymmetric Fermi surfaces. ${ }^{24-26}$ While constructive renormalization (see, e.g., Ref. 27 for an introduction) is based on the $\mathrm{RG}$ ideas, the way in which it implements these ideas is slightly different with respect to other more standard schemes, the main advantages being that the resulting method is (i) exact, in the sense that it does not need any relativistic approximation or continuum limit and it allows us to keep the full lattice structure of the problem; (ii) nonperturbative, in the sense that it involves expansions whose convergence can be mathematically proved.

The Hamiltonian of the 2D Hubbard model on the honeycomb lattice at half filling in second-quantized form is given by

$$
\begin{aligned}
H_{\Lambda}= & -\sum_{\substack{\vec{x} \in \Lambda \\
i=1,2,3}} \sum_{\sigma=\uparrow \downarrow}\left(a_{\vec{x}, \sigma^{+}}^{+} b_{\vec{x}+\vec{\delta}_{i}, \sigma}+b_{\vec{x}+\vec{\delta}_{i}, \sigma^{\prime}, a^{-}}^{+}\right)+U \sum_{\substack{\vec{x} \in \Lambda \\
i=1,2,3}} \sum_{\sigma, \sigma^{\prime}} \\
& \times\left(a_{\vec{x}, \sigma^{\prime}}^{+} a_{\vec{x}, \sigma}^{-}-\frac{1}{2}\right)\left(b_{\vec{x}+\vec{\delta}_{i}, \sigma^{\prime}}^{+} b_{\vec{x}+\vec{\delta}_{i}, \sigma^{\prime}}^{-}-\frac{1}{2}\right),
\end{aligned}
$$

where $\Lambda$ is a periodic triangular lattice with basis $\vec{a}_{1,2}$ $=\frac{1}{2}( \pm \sqrt{3}, 3)$ and the nearest-neighbor vectors $\vec{\delta}_{i}$ are defined as $\vec{\delta}_{1}=(0,1)$ and $\vec{\delta}_{2,3}=\frac{1}{2}( \pm \sqrt{3},-1)$. The creation and annihilation fermionic operators with spin index $\sigma=\uparrow \downarrow$, $a_{\vec{x}, \sigma}^{ \pm}, \quad b_{\vec{x}+\vec{\delta}_{i}, \sigma}^{ \pm}$, satisfy periodic boundary conditions in $\vec{x}$. The choice of the interaction is done only for definiteness (it is the simplest one for which the anisotropy of the charge velocity is visible at first order in renormalized perturbation theory) but our results are valid for a generic short-range density-density interaction.

We introduce the two-component fermionic operators $\psi_{\vec{x}, \sigma}^{ \pm}=\left(a_{\vec{x}, \sigma}^{ \pm}, b_{\vec{x}+\vec{\delta}_{1}, \sigma}^{ \pm}\right)$and $\psi_{\mathbf{x}, \sigma}^{ \pm}=e^{H_{\Lambda} x_{0}} \psi_{\vec{x}, \sigma}^{ \pm} e^{-H_{\Lambda} x_{0}}$ with $\mathbf{x}=\left(x_{0}, \vec{x}\right)$. If $\langle\cdot\rangle=\lim _{\beta,|\Lambda| \rightarrow \infty} \operatorname{Tr}\left\{e^{-\beta H_{\Lambda}} \mathbf{T}\{\cdot\}\right\} / \operatorname{Tr}\left\{e^{-\beta H_{\Lambda}}\right\}$, with $\mathbf{T}$ as the fermionic time-ordering operator, the zero-temperature $2 n$-point Schwinger functions are defined as $\left\langle\prod_{i=1}^{n} \psi_{\mathbf{x}_{i}, \sigma_{i}}^{-} \psi_{\mathbf{y}_{i}, \sigma_{i}^{\prime}}^{+}\right\rangle$. In the noninteracting $U=0$ case, the Fourier transform of the twopoint Schwinger function is given by

$$
\hat{S}_{0}(\mathbf{k})=\left.\left\langle\hat{\psi}_{\mathbf{k}, \sigma}^{-} \hat{\psi}_{\mathbf{k}, \sigma}^{+}\right\rangle\right|_{U=0}=\left(\begin{array}{cc}
-i k_{0} & -v^{*}(\vec{k}) \\
-v(\vec{k}) & -i k_{0}
\end{array}\right)^{-1},
$$

with $\hat{\psi}_{\mathbf{k}, \sigma}^{ \pm}=\int_{-\beta / 2}^{\beta / 2} d x_{0} \sum_{\vec{x} \in \Lambda} e^{\mp i \mathbf{k x}} \psi_{\mathbf{x}, \sigma}^{ \pm}$and $v(\vec{k})=\sum_{i=1}^{3} e^{i \vec{k}\left(\vec{\delta}_{i}-\vec{\delta}_{1}\right)}$. $\hat{S}_{0}(\mathbf{k})$ is singular at the Fermi points $\mathbf{p}_{F}^{ \pm}=\left(0, \vec{p}_{F}^{ \pm}\right)$, where $\vec{p}_{F}^{ \pm}$ $=\left( \pm \frac{2 \pi}{3 \sqrt{3}}, \frac{2 \pi}{3}\right)$. Close to $\vec{p}_{F}^{ \pm}, v\left(\vec{k}^{\prime}+\vec{p}_{F}^{ \pm}\right) \simeq(3 / 2)\left( \pm k_{1}^{\prime}+i k_{2}^{\prime}\right)$, so that the free Schwinger function is asymptotically the same as that of the massless Dirac fermions in $2+1$ dimensions.

The density operator is defined as $\hat{\rho}_{\mathbf{p}}$ $=(\beta|\Lambda|)^{-1} \sum_{\mathbf{k}, \sigma} \hat{\psi}_{\mathbf{k}, \sigma}^{+} \hat{\psi}_{\mathbf{k}-\mathbf{p}, \sigma}^{-}$and the definition of the current, for $U=0$, is obtained from the equation $d \rho_{\mathbf{x}} / d x_{0}=\left[H_{\Lambda}, \rho_{\mathbf{x}}\right]$; in fact, the latter, for small $\mathbf{p}$, assumes the form of a continuity equation provided that the current is chosen as $\hat{J}_{\mathbf{p}, i}$
$=(\beta|\Lambda|)^{-1} \sum_{\mathbf{k}, \sigma} \hat{\psi}_{\mathbf{k}, \sigma}^{+} \sigma_{i} \hat{\psi}_{\mathbf{k}-\mathbf{p}, \sigma}^{-}$, where $i=1,2$, with $\sigma_{1}$ and $\sigma_{2}$ as the first two Pauli matrices. The continuity equation implies the validity of an asymptotic Ward identity; defining $\mathbf{k}^{\prime}=\mathbf{k}-\mathbf{p}_{F}^{ \pm}$, if $\mathbf{k}^{\prime}, \mathbf{p}$, and $\mathbf{k}^{\prime}-\mathbf{p}$ are small and of the same order of magnitude, ${ }^{28}$

$$
\begin{aligned}
& \left\langle\left(i p_{0} \hat{\rho}_{\mathbf{p}} \pm(3 / 2) p_{1} \hat{J}_{\mathbf{p}, 1}+(3 / 2) p_{2} \hat{J}_{\mathbf{p}, 2}\right) ; \hat{\psi}_{\mathbf{k}, \sigma}^{-} \hat{\psi}_{\mathbf{k}-\mathbf{p}, \sigma}^{+}\right\rangle \\
& \simeq\left[\left\langle\hat{\psi}_{\mathbf{k}, \sigma}^{-} \hat{\psi}_{\mathbf{k}, \sigma}^{+}\right\rangle-\left\langle\hat{\psi}_{\mathbf{k}-\mathbf{p}, \sigma}^{-} \hat{\psi}_{\mathbf{k}-\mathbf{p}, \sigma}^{+}\right\rangle\right] .
\end{aligned}
$$

When the interaction is present, the Schwinger functions are not exactly computable anymore; however, quite remarkably, they can be computed in terms of a convergent renormalized perturbative series, and their long-distance asymptotic properties can be rigorously derived, as summarized by the following theorem.

THEOREM 1. There exists a constant $U_{0}>0$ such that if $|U| \leq U_{0}$, the specific ground-state energy and the zerotemperature Schwinger functions of model (1) are analytic functions of $U$. The Fourier transform of the two-point Schwinger function $S(\mathbf{k})$ is singular only at $\mathbf{k}=\mathbf{p}_{F}^{ \pm}$and, close to the singularity, it can be written as ${ }^{28}$

$$
S(\mathbf{k}) \simeq \frac{1}{Z}\left(\begin{array}{cc}
-i k_{0} & v_{F}\left(\mp k_{1}^{\prime}+i k_{2}^{\prime}\right) \\
v_{F}\left(\mp k_{1}^{\prime}-i k_{2}^{\prime}\right) & -i k_{0}
\end{array}\right)^{-1},
$$

where $Z=Z(U)$ and $v_{F}=v_{F}(U)$ are analytic functions of $U$, such that $Z=1+O\left(U^{2}\right)$ and $v_{F}=3 / 2+b U+O\left(U^{2}\right)$, with

$$
b=\left.\int_{B_{1}} \frac{d \vec{k}}{2\left|B_{1}\right|} \frac{v(\vec{k})}{|v(\vec{k})|} \partial_{p_{1}} v(\vec{p}-\vec{k})\right|_{\vec{p}=\vec{p}_{F}^{+}}=0.511 \ldots,
$$

and $B_{1}$ as the first Brillouin zone.

The result summarized in theorem 1 says that the interaction does not qualitatively change the asymptotic behavior of the two-point Schwinger function close to the Fermi points; the effect of the interaction is essentially to change, by a finite amount, the wave-function renormalization and the Fermi velocity. This implies that the interacting correlations decay as fast as in the noninteracting case, and therefore, the presence of quantum instabilities in the ground state, such as Néel or superconducting long-range order, is rigorously excluded at half filling and weak coupling, together with the possibility of a mass generation.

Note also that in the presence of the interaction, the Fermi velocity remains the same in the two coordinate directions even though the model does not display $90^{\circ}$ discrete rotational symmetry but rather a $120^{\circ}$ rotational symmetry; such a remarkable property can be easily checked at first order [replacing $\partial_{p_{1}}$ by $-i \partial_{p_{2}}$ in Eq. (5), the same result is found]; for a proof at all orders in the convergent expansion for $v_{F}$, see below. The isotropy of the Fermi velocity implies the isotropy of the Dirac cones at low energies.

THEOREM 2. For $|U| \leq U_{0}$, if $\mathbf{k}^{\prime}$, $\mathbf{p}$, and $\mathbf{k}^{\prime}-\mathbf{p}$ are small and of the same order of magnitude, ${ }^{28}$ then

$$
\begin{gathered}
\left\langle\left(i p_{0} \hat{\rho}_{\mathbf{p}} \pm v_{1} p_{1} \hat{J}_{\mathbf{p}, 1}+v_{2} p_{2} \hat{J}_{\mathbf{p}, 2}\right) ; \hat{\psi}_{\mathbf{k}, \sigma}^{-} \hat{\psi}_{\mathbf{k}-\mathbf{p}, \sigma}^{+}\right\rangle \\
\simeq\left[\left\langle\hat{\psi}_{\mathbf{k}, \sigma}^{-} \psi_{\mathbf{k}, \sigma}^{+}\right\rangle-\left\langle\hat{\psi}_{\mathbf{k}-\mathbf{p}, \sigma}^{-} \hat{\psi}_{\mathbf{k}-\mathbf{p}, \sigma}^{+}\right\rangle\right],
\end{gathered}
$$

where the charge velocity $v_{1,2}=3 / 2+O(U)$ is analytic in $U$ 


$$
\begin{aligned}
& \text { and } v_{1}-v_{2}=a U+O\left(U^{2}\right) \text {, with } \\
& \qquad a=\frac{3}{4} \int_{B_{1}} \frac{d \vec{k}}{\left|B_{1}\right|} \frac{v^{2}(\vec{k})}{|v(\vec{k})|^{3}} v^{*}\left(\vec{k}-\vec{p}_{F}^{+}\right)=-0.03165 \ldots
\end{aligned}
$$

Theorem 2 says that in the presence of interactions, a new Ward identity, different from the noninteracting one, is verified, the main difference with respect to Eq. (3) being that the charge velocity $\left(v_{1}, v_{2}\right)$ is interaction dependent and different from the Fermi velocity. Remarkably, its two components are different; this anisotropy is related to the presence of the lattice, that is, to the irrelevant terms in a RG sense, which are not neglected in our exact scheme. In fact, if we replace $v\left(\vec{k}^{\prime}+\vec{p}_{F}^{ \pm}\right)$by its asymptotic expression $(3 / 2)\left( \pm k_{1}^{\prime}\right.$ $\left.+i k_{2}^{\prime}\right)$, the anisotropy coefficient defined by Eq. (7) vanishes exactly (and so do the higher-order corrections). We remark that while theorems 1 and 2 refer to the case of short-range interactions, the conclusions concerning the symmetry of the Fermi and charge velocities remain true, as statements at all orders, even for the case of unscreened Coulomb interactions.

We now sketch the proof of the two theorems above (for a detailed proof we refer to Ref. 29). The starting point is the well-known representation of the ground-state energy in terms of a Grassmann functional integral $e_{0}$ $=\lim _{\beta,|\Lambda| \rightarrow \infty}(\beta|\Lambda|)^{-1} \log \int P(d \psi) \exp \{\mathcal{V}(\psi)\}$, where $P(d \psi)$ is the Grassmann Gaussian integration with propagator $\hat{S}_{0}(\mathbf{k})$, see Eq. (2), and $\mathcal{V}(\psi)$ is the quartic interaction Eq. (1). One can compute $e_{0}$ by expanding the exponential $\exp \{\mathcal{V}(\psi)\}$ in Taylor series in $U$ and naively integrating term by term the Grassmann monomials, using the Wick rule; however, by such procedure, it is very difficult to take into account the cancellations present in the perturbative series. The bounds obtained by this "simple" procedure are nonuniform in $\beta$, and they do not allow one to take the thermodynamic and zero-temperature limits. Therefore, we set up an iterative procedure for the computation of $e_{0}$, based on (Wilsonian) $\mathrm{RG}$ and involving nontrivial resummations of the perturbative series.

The first step is to decompose the propagator $\hat{S}_{0}(\mathbf{k})$ as a sum of propagators supported close to the two Fermi points and more and more singular in the infrared region, labeled by a quasiparticle index $\omega= \pm$ (labeling the Fermi points) and by an integer $h \leq 0$ so that $\hat{S}_{0}(\mathbf{k})=\sum_{h \leq 1}^{\omega= \pm} \hat{g}_{\omega}^{(h)}\left(\mathbf{k}-\mathbf{p}_{F}^{\omega}\right)$, with $\hat{g}_{\omega}^{(h)}$ supported on quasimomenta of scale $2^{h}$ and, on the support, of size $\left\|\hat{g}_{\omega}^{(h)}\right\| \sim 2^{-h}$. At this point, we compute $e_{0}$ by iteratively integrating the propagators $\hat{g}^{(0)}, \hat{g}^{(-1)}, \ldots$. After each integration step we rewrite

$$
e_{0}=F_{h}+\lim _{\beta,|\Lambda| \rightarrow \infty} \frac{1}{\beta|\Lambda|} \log \int \prod_{\omega= \pm} P\left(d \psi_{\omega}^{(\leq h)}\right) e^{\mathcal{V}^{(h)}\left(\psi^{(\leq h)}\right)},
$$

where $P\left(d \psi_{ \pm}^{(\leq h)}\right)$ is the Grassmannian quadratic integration with propagator given by

$$
g_{ \pm}^{(\leq h)}\left(\mathbf{k}^{\prime}\right) \simeq \frac{\chi_{h}\left(\mathbf{k}^{\prime}\right)}{Z_{h}}\left(\begin{array}{cc}
-i k_{0} & c_{h}\left(\mp k_{1}^{\prime}+i k_{2}^{\prime}\right) \\
c_{h}\left(\mp k_{1}^{\prime}-i k_{2}^{\prime}\right) & -i k_{0}
\end{array}\right)^{-1},
$$

where $\chi_{h}^{-1}\left(\mathbf{k}^{\prime}\right)$ is a smooth compact support function nonva- nishing only for $\left|\mathbf{k}^{\prime}\right| \leq 2^{h} ; \mathcal{V}^{(h)}$ is the effective potential, a sum of monomials of arbitrary order, with kernels that are analytic functions of $U$; analyticity it is a very nontrivial property obtained by exploiting anticommutativity properties of Grassmann variables via Gram inequality for determinants. The scaling dimensions of the kernels with $n_{e}$ external lines are equal to $3-n_{e}$, modulo an additional dimensional gain, following from the fact that all kernels with $\geq 4$ external lines are irrelevant in a RG sense (see Ref. 29, theorem $2)$. The kernels $\hat{W}_{2}^{(h)}\left(\mathbf{k}^{\prime}\right)$ with $n_{e}=2$, which are linearly relevant, can be inserted step by step in the Gaussian integration, thanks to the fact that they have the same reality and symmetry properties as the free quadratic action; in particular, it is found that $\hat{W}_{2}^{(h)}(\mathbf{0})=0$ and

$$
\mathbf{k}^{\prime} \partial_{\mathbf{k}^{\prime}} \hat{W}_{2}^{(h)}(\mathbf{0})=\left(\begin{array}{cc}
-i z_{h} k_{0} & \delta_{h}\left(\mp k_{1}^{\prime}+i k_{2}^{\prime}\right) \\
\delta_{h}\left(\mp k_{1}^{\prime}-i k_{2}^{\prime}\right) & -i z_{h} k_{0}
\end{array}\right)
$$

for suitable real constants $z_{h}$ and $\delta_{h}$. Note that $\mp k_{1}^{\prime}$ and $i k_{2}^{\prime}$ are multiplied by the same constant $\delta_{h}$, which is quite remarkable; see Ref. 29, lemma 2 for a proof. Iterating the procedure above, we find recursive equations for $Z_{h}$ and $c_{h}$; in the $h \rightarrow-\infty$ limit, the two running coupling constants converge to values $Z_{-\infty}=Z=Z(U)$ and $c_{-\infty}=v_{F}=v_{F}(U)$, which are close to their unperturbed values and are analytic in $U$ (again, thanks to the fact that all subdiagrams with $n_{e} \geq 4$ are irrelevant in a RG sense). This completes the discussion concerning the analyticity of $e_{0}$. A similar discussion allows us to prove the analyticity of the Schwinger functions and Eq. (4), see Ref. 29, Sec. IIID.

Let us now discuss a sketch of the proof of theorem 2 . We perform a multiscale analysis similar to the one sketched above, with $\mathcal{V}(\psi)$ replaced by $\mathcal{V}(\psi)-\mathcal{B}(\phi, J)$, with $\mathcal{B}(\phi, J)=\left(\phi^{+}, \psi^{-}\right)+\left(\psi^{+}, \phi^{-}\right)+\sum_{\mu=0}^{2}\left(J_{\mu}, j_{\mu}\right), \phi_{\mathbf{x}, \sigma}^{ \pm}$as two external Grassmann fields, $J_{\mathbf{x}, \mu}$ as an external commuting field, and $j_{\mathbf{x}, \mu}$ as the current [here $j_{\mathbf{x}, 0}=\rho_{\mathbf{x}}$, with $\rho$ as the density, see the lines preceding Eq. (3)].

The iterative integration procedure described above, in this case, produces, besides the effective potential $\mathcal{V}^{(h)}$, new terms involving the external fields. In particular, at scale $h$, the effective source term is given by $\Sigma_{\mu, \mu^{\prime}} Z_{\mu, \mu^{\prime}}^{(h)}\left(J_{\mu}, j_{\mu^{\prime}}^{(\leq h)}\right)$, with $Z_{\mu, \mu^{\prime}}=\delta_{\mu, \mu^{\prime}} Z_{\mu}$, by the discrete invariance symmetries of the model, see Ref. 29, lemma 1 . A crucial remark is that while in a relativistic quantum field theory (QFT) $Z_{\mu}^{(h)}$ is $\mu$ independent, here it is not, because the relativistic symmetry is broken by the presence of the underlying lattice (i.e., by the irrelevant terms in the fermionic action). We find that in the limit $h \rightarrow-\infty, Z_{\mu}^{(h)} \rightarrow Z_{\mu}=Z_{\mu}(U)$, which are analytic functions of $U$, with $Z_{0}=Z=1+O\left(U^{2}\right)$ and $Z_{2}-Z_{1}=(2 a / 3) U$ $+O\left(U^{2}\right)$, where $a$ is given in Eq. (7). See Fig. 1 .

Using the fact that all kernels with four or more external lines are irrelevant, we find that, for small $\mathbf{k}^{\prime}, \mathbf{k}^{\prime}-\mathbf{p}$, and $\mathbf{p}$,

$$
\left\langle\hat{J}_{\mathbf{p}, \mu} ; \hat{\psi}_{\mathbf{k}-\mathbf{p}, \sigma}^{-} \psi_{\mathbf{k}, \sigma}^{+}\right\rangle \simeq Z_{\mu} S(\mathbf{k}) \sigma_{\mu} S(\mathbf{k}-\mathbf{p}), \quad \mu=0,1,2,
$$

with $\sigma_{0}=1$ and $S(\mathbf{k})$ as the interacting two-point Schwinger function in Eq. (4). The combination $S(\mathbf{k}) \sigma_{\mu} S(\mathbf{k}+\mathbf{p})$ is as- 

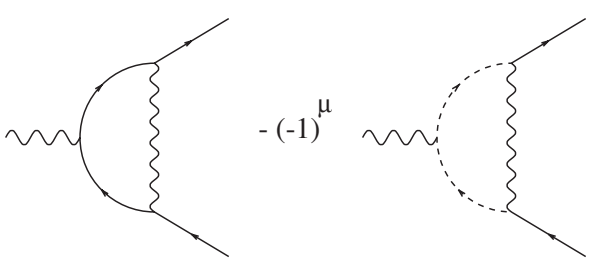

FIG. 1. The two graphs contributing at first order to $Z_{\mu}$, where $\mu=1,2$. The full line corresponds to a diagonal propagator and the dotted line corresponds to an off-diagonal one. Note that the two graphs appear with a different sign, depending on the value of $\mu$. The second graph is vanishing in the continuum approximation $v(\vec{k}) \simeq(3 / 2)\left( \pm k_{1}^{\prime}+i k_{2}^{\prime}\right)$.

ymptotically the same as the vertex of a relativistic QFT with wave-function renormalization $Z$ and "speed of light" equal to $v_{F}$; therefore, it satisfies a relativistic Ward identity (WI), relating it to the derivative of $S(\mathbf{k})$,

$$
\begin{gathered}
S(\mathbf{k})\left[i p_{0}+v_{F}\left( \pm p_{1} \sigma_{1}+p_{2} \sigma_{2}\right)\right] S(\mathbf{k}-\mathbf{p}) \\
\simeq \frac{1}{Z}[S(\mathbf{k})-S(\mathbf{k}-\mathbf{p})] .
\end{gathered}
$$

Combining Eqs. (11) and (12), and recalling that, by symmetry, $Z=Z_{0}$, we get Eq. (6) with $v_{1,2}=v_{F} Z_{0} / Z_{1,2}$.

In conclusion, we analyzed the properties of a single-layer graphene at half filling, described by a Hubbard model on the honeycomb lattice, going beyond the approaches based on finite-order truncations and relativistic approximations (previous analyses taking into account lattice effects were focused on doped rather than on half-filled graphene, see, e.g.,
Refs. 11 and 30-32). In the case of short-range interactions, we proved the analyticity of the theory at weak coupling; this gives a rigorous confirmation to the belief (see, e.g., Ref. 19) that nonperturbative effects such as quantum instabilities or the opening of a mass gap can be possibly present only at large values of the coupling. We proved that the Fermi velocity and the Dirac cones at low energies are isotropic, in agreement with observations based on angle-resolved photoemission spectroscopy; ${ }^{5,6}$ previous analyses based on relativistic approximations were inconclusive in this respect since the (previously neglected) symmetry-breaking terms due to the lattice produce a renormalization of the Fermi velocity, which could in principle be anisotropic. This is by no means just an academic possibility; indeed, while this anisotropy effect is not visible in the Fermi velocity, we show that it is observable in other quantities, such as the charge velocity appearing in the Ward identities, which turns out to be asymmetric in the two coordinate directions. This asymmetry was previously unnoticed and it may be detected in future experiments. Finally, we stress that the assumption of local or short-range interaction plays a crucial role in our analysis; the unscreened Coulomb interactions is marginal instead of irrelevant in the RG sense, and its effect on the physical properties can be in principle much more relevant. The unscreened Coulomb interactions have been studied up to now mainly in the relativistic approximation and at lowest perturbative orders, starting from Refs. 9 and 10, and we believe that going beyond such approximations will give a definite answer to the question of the role of the interactions in the properties of real graphene.

We thank G. Benfatto for many useful discussions. *giuliani@mat.uniroma3.it

†mastropi@axp.mat.uniroma2.it

${ }^{1}$ K. S. Novoselov et al., Science 306, 666 (2004).

${ }^{2}$ P. R. Wallace, Phys. Rev. 71, 622 (1947).

${ }^{3}$ K. S. Novoselov et al., Nature (London) 438, 197 (2005).

${ }^{4}$ A. K. Geim and K. S. Novoselov, Nature Mater. 6, 183 (2007).

${ }^{5}$ S. Y. Zhou et al., Nat. Phys. 2, 595 (2006).

${ }^{6}$ A. Bostwick et al., Nat. Phys. 3, 36 (2007).

${ }^{7}$ G. W. Semenoff, Phys. Rev. Lett. 53, 2449 (1984).

${ }^{8}$ R. Jackiw and S.-Y. Pi, Phys. Rev. Lett. 98, 266402 (2007).

${ }^{9}$ J. Gonzalez et al., Nucl. Phys. B 424, 595 (1994).

${ }^{10}$ J. Gonzalez et al., Phys. Rev. B 63, 134421 (2001).

${ }^{11}$ S. Das Sarma et al., Phys. Rev. B 75, 121406(R) (2007).

${ }^{12}$ C. H. Park et al., Phys. Rev. Lett. 99, 086804 (2007).

${ }^{13}$ E. G. Mishchenko, Phys. Rev. Lett. 98, 216801 (2007).

${ }^{14}$ D. E. Sheehy and J. Schmalian, Phys. Rev. Lett. 99, 226803 (2007).

${ }^{15}$ V. N. Kotov et al., Phys. Rev. B 78, 035119 (2008).

${ }^{16}$ I. F. Herbut et al., Phys. Rev. Lett. 100, 046403 (2008).

${ }^{17}$ A. H. Castro Neto et al., Rev. Mod. Phys. 81, 109 (2009).

${ }^{18}$ V. P. Gusynin et al., Int. J. Mod. Phys. B 21, 4611 (2007).
${ }^{19}$ I. Herbut et al., Phys. Rev. B 79, 085116 (2009).

${ }^{20}$ I. F. Herbut, Phys. Rev. Lett. 97, 146401 (2006).

${ }^{21}$ D. V. Khveshchenko, J. Phys.: Condens. Matter 21, 075303 (2009).

${ }^{22}$ E. H. Lieb, Phys. Rev. Lett. 62, 1201 (1989).

${ }^{23} \mathrm{G}$. Benfatto and V. Mastropietro, Commun. Math. Phys. 258, 609 (2005).

${ }^{24}$ M. Disertori and V. Rivasseau, Phys. Rev. Lett. 85, 361 (2000).

${ }^{25}$ G. Benfatto et al., Ann. Henri Poincare 7, 809 (2006).

${ }^{26}$ J. Feldman et al., Commun. Math. Phys. 247, 1 (2004).

${ }^{27}$ V. Mastropietro, Non-Perturbative Renormalization (World Scientific, Singapore, 2008).

${ }^{28}$ The symbol $\simeq$ means that the ratio of the left-hand side to the right-hand side is equal to $1+R$, with $R$ as a matrix computed in terms of a convergent series and satisfying $\|R\| \leq C_{\theta}\left|\mathbf{k}-\mathbf{p}_{F}^{ \pm}\right|^{\theta}$ for any $0<\theta<1$ and a suitable constant $C_{\theta}$.

${ }^{29}$ A. Giuliani and V. Mastropietro, arXiv:0811.1881 (unpublished).

${ }^{30}$ R. Roldan et al., Phys. Rev. B 77, 115410 (2008).

${ }^{31}$ B. Valenzuela and M. Vozmediano, New J. Phys. 10, 113009 (2008).

${ }^{32}$ M. Polini et al., Solid State Commun. 143, 58 (2007). 